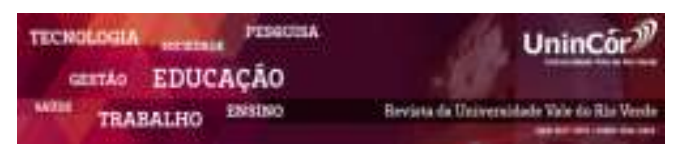

Revista da Universidade Vale do Rio Verde ISSN: 1517-0276 / EISSN: 2236-5362 Vol. $16 \mid$ n. 2 | Ano 2018

Jocelaine Dall'agnol Universidade Estadual do Centro-Oeste johdallagnol@hotmail.com

Liégi Tajana Ferranti Universidade Estadual do Centro-Oeste ly_ferranti@hotmail.com

Letícia Claudy

Universidade Estadual do Centro-Oeste leticiaclaudy@hotmail.com

Gabriel Cecchin

Universidade Estadual do Centro-Oeste gabri_el135@hotmail.com

Herta Stutz Dalla Santa

Universidade Estadual do Centro-Oeste hdalsanta@yahoo.com.br

Bruna Menegassi Universidade Estadual do Centro-Oeste brunamenegassi.nut@gmail.com

Daiana Novello Universidade Estadual do Centro-Oeste nutridai@gmail.com

\section{AVALIAÇ̃̃o FÍSICO-QUíMICA DE PÃO BRANCO E PÃO INTEGRAL: COMPARAÇÃO COM O RÓTULO NUTRICIONAL}

\begin{abstract}
RESUMO
As informações contidas nos rótulos dos alimentos possibilitam conhecer a origem dos alimentos, assim como sua constituição, sendo que a saúde do indivíduo pode ser prejudicada quando os rótulos não contem informações fidedignas sobre sua composição. O objetivo deste estudo foi avaliar a composição físico-química de pães brancos e integrais, visando sua comparação com rótulos nutricionais apresentados nestes produtos. Realizou-se a análise laboratorial de 6 amostras de pães, sendo 3 marcas de pão branco e 3 de pão integral, comparadas com seus respectivos rótulos. Observou-se que a maioria das amostras avaliadas estava em inconformidade com o rótulo dos alimentos e com a tabela de composição química de alimentos. Além disso, foram encontradas inadequações na variação tolerada pela legislação vigente, principalmente nas amostras de pão branco. Dessa forma, torna-se indispensável aos fabricantes e indústrias um maior investimento nesta área, já que é direito do consumidor conhecer a composição do alimento que está adquirindo.
\end{abstract}

Palavras-chave: Cereais. Nutrientes. Informação nutricional.

\section{PHYSICO-CHEMICAL EVALUATION OF WHITE BREAD AND INTEGRAL BREAD: COMPARISON WITH NUTRITION LABEL}

\begin{abstract}
The information contained on food labels allow to know the origin of food, as well as its constitution, and the health of the individual may be impaired when the labels do not contain reliable information on their composition. The objective of this study was to evaluate the physico-chemical composition of white and whole grain breads, for their comparison with nutrition labels shown in these products. We conducted laboratory analysis of 6 samples of breads, 3 white bread brands and 3 whole wheat bread, compared with their respective labels. It was observed that most of the samples was in disagreement with the labeling of food and the food composition table. In addition, inadequacies were found in the range tolerated by the current legislation, especially in white bread samples. Thus, it is essential for manufacturers and industries increased investment in this area, as is the consumer's right to know about the composition that is acquiring.
\end{abstract}

Keywords: Cereals. Nutrients. Nutrition information.

Recebido em: 10/01/2018 - Aprovado em: 25/07/2018 - Disponibilizado em: 15/12/2018 


\section{INTRODUÇÃO}

Toda descrição destinada a informar ao consumidor sobre as propriedades nutricionais de um alimento é denominada rotulagem nutricional. Compreende as declarações de valor energético e nutrientes bem como a declaração de propriedades nutricionais (BRASIL, 2003), sendo também conhecidas por informação nutricional e informação nutricional complementar, respectivamente.

Apesar dos consumidores encontrarem dificuldades em interpretar as informações contidas nos rótulos dos alimentos e nas tabelas de informações nutricionais (BENDINO et al., 2012), elas podem influenciar positiva ou negativamente na escolha do consumidor. Isso, porque são importantes em relação aos parâmetros de segurança e qualidade, além de imprescindíveis em casos de restrições e dietas alimentares (LOBANCO et al., 2009).

A rotulagem nutricional de alimentos tornou-se obrigatória no Brasil em 1999, com a criação da Agência Nacional de Vigilância Sanitária (ANVISA) (LOBANCO et al., 2009). Entre as resoluções da diretoria colegiada (RDC), referentes à rotulagem de alimentos industrializados no Brasil, está a RDC n. 360 que estabelece, dentre outras especificações, a declaração obrigatória nos rótulos de alimentos industrializados de: valor energético, teor de carboidratos, proteínas, gorduras totais, gorduras saturadas, gorduras trans, fibra alimentar e sódio (BRASIL, 2003).

Para a elaboração da informação nutricional, a ANVISA autoriza a obtenção dos dados de nutrientes por meio de análises físicoquímicas ou por cálculos teóricos baseados na fórmula do produto, obtidos de valores de tabelas de composição de alimentos ou fornecidos pelos fabricantes de matérias-primas (LOBANCO et al., 2009). As análises físico-químicas, apesar de fornecerem resultados mais fidedignos dos nutrientes, não são preferidas pelos fabricantes, entre outros fatores, pelo seu alto custo, quando comparadas às outras formas de obtenção desses dados. Nesse sentido, por exemplo, valores de tabelas de composição de alimentos, utilizados por fabricantes de matérias-primas, podem ter sofrido arredondamentos que terão novas alterações para a elaboração da informação nutricional do produto final. Assim, o uso corrente dessa prática pode resultar em uma cadeia de erros que, ao final, implicarão em valores de informação nutricional sub ou superestimados e que não corresponderão à verdadeira composição do produto. Dessa forma, quando as informações nutricionais não apresentam valores fidedignos acerca dos nutrientes declarados, o indivíduo pode ser prejudicado. Além disso, o mesmo pode estar sendo lesado economicamente. Apesar da ANVISA admitir uma tolerância de 20\% (para mais ou para menos) com relação aos valores de nutrientes declarados no rótulo, o cenário brasileiro de atendimento à legislação de rotulagem nutricional ainda é precário (CÂMARA et al., 2008).

Os pães são fontes importantes de carboidratos, proteínas e vitaminas (MENEZES et al., 2009) e, por isso, são produtos muito apreciados pela população, apresentando elevado consumo mundial. Podem ser produzidos a partir de diversos cereais e ingredientes, fato que dificulta, muitas vezes, uma análise precisa de sua composição nutricional. Nesse contexto, o presente trabalho teve por objetivo avaliar a composição físico- 
química de pães branco e integral, visando à comparação com a tabela de informação nutricional informada nos produtos.

\section{MÉTODOS}

Foram analisadas 3 marcas de pão branco (denominadas $\mathrm{Pb} 1, \mathrm{~Pb} 2$ e $\mathrm{Pb} 3$ ) e 3 de pão integral (Pi1, Pi2 e Pi3). Os produtos foram adquiridos em um supermercado local da cidade de Guarapuava, PR, dentro do prazo de validade e armazenados conforme especificações do fabricante. Para proceder as análises físico-química, 5 fatias de cada marca de pão foram devidamente trituradas em multiprocessador (Wallita ${ }^{\circledR}, \quad$ Brasil), homogeneizadas e armazenadas em recipientes hermeticamente fechados.

As análises físico-químicas dos produtos foram realizadas, em triplicata, nos Laboratórios de Análise de Alimentos e de Processos, ambos do Departamento de Engenharia de Alimentos da Universidade Estadual do Centro-Oeste (UNICENTRO). Foram determinadas: umidade por dessecação em estufa a $105{ }^{\circ} \mathrm{C}$ até peso constante (IAL, 2008); proteínas pelo método de micro Kjeldahl, determinando-se o nitrogênio total e utilizando-se o fator de conversão de 6,25 para o cálculo do teor de proteínas (IAL, 2008) e; lipídeos, seguindo metodologia proposta por Bligh e Dyer (1959). Os carboidratos foram determinados através de cálculo teórico (por diferença) dos resultados das triplicatas (IAL, 2008), conforme fórmula: $\%$ carboidratos $=100$ $-(\%$ umidade $+\%$ proteína $+\%$ lipídios $+\%$ cinzas). O valor calórico total (kcal) foi calculado em relação a 100 g de amostra, utilizando-se os valores de Atwater (ou calor de combustão) para gordura (9 kcal/ grama), proteína $(4,02 \mathrm{kcal} /$ grama) e carboidratos $(3,87 \mathrm{kcal} /$ grama $)$ (ATWATER; WOODS, 1896).

O percentual de Valor Diário de Referência (VD) foi calculado com base na recomendação da Dietary Reference Intakes (2005) para adultos, considerando que uma dieta de $2.000 \mathrm{kcal}$ é composta em média por $60 \%$ de carboidratos, $15 \%$ de proteínas e $25 \%$ de lipídios totais.

Os resultados de proteínas e lipídeos obtidos através de análises físico-químicas, bem como os resultados de carboidratos e calorias obtidos através de cálculos foram comparados com aqueles declarados nas tabelas de informações nutricionais dos produtos. Os resultados de umidade e cinzas das análises laboratoriais foram comparados aos valores disponíveis na Tabela de Composição Química de Alimentos (TACO, 2011), já que a informação nutricional de produtos embalados não dispõe desses valores.

Os resultados foram avaliados estatisticamente com auxílio do software Statgraphics Plus ${ }^{\circledR}$, versão 5.1, através de análise de médias e desvio padrão, frequências e porcentagens.

\section{RESULTADOS E DISCUSSÃO}

$\mathrm{Na}$ Tabela 1 estão descritos os teores médios da composição físico-química, comparados aos valores declarados nas tabelas de informações nutricionais dos produtos e/ou Tabela de Composição Química de Alimentos. 
Tabela 1 - Valores obtidos em análises laboratoriais e declarados nas tabelas de informações nutricionais e/ou tabela de composição química de alimentos (porção de $100 \mathrm{~g}$ ), dos pães brancos $(\mathrm{Pb})$ e integrais $(\mathrm{Pi})$

\begin{tabular}{|c|c|c|c|c|}
\hline Parâmetros & Valor analisado & VD $(\%)^{*}$ & Valor tabelado & VD $(\%)^{*}$ \\
\hline \multicolumn{5}{|l|}{ Pb1 } \\
\hline Umidade (\%) & $34,29 \pm 0,23$ & - & $26,00^{\mathrm{a}}$ & - \\
\hline Cinzas $(\%)$ & $1,83 \pm 0,13$ & - & $1,50^{\mathrm{a}}$ & - \\
\hline Proteínas $(\%)$ & $12,40 \pm 0,20$ & 16,50 & $14,00^{\mathrm{b}}$ & 11,00 \\
\hline Lipídeos (\%) & $1,24 \pm 0,19$ & 2,25 & $2,00^{\mathrm{b}}$ & 1,00 \\
\hline Carboidratos $(\%)$ & $50,23 \pm 0,32$ & 16,70 & $86,00^{\mathrm{b}}$ & 11,00 \\
\hline Calorias (kcal) & $261,83 \pm 2,15$ & 13,00 & $192,00^{\mathrm{b}}$ & 9,60 \\
\hline \multicolumn{5}{|l|}{ Pb2 } \\
\hline Umidade (\%) & $31,51 \pm 0,30$ & - & $26,00^{\mathrm{a}}$ & - \\
\hline Cinzas $(\%)$ & $1,86 \pm 0,13$ & - & $1,50^{\mathrm{a}}$ & - \\
\hline Proteínas (\%) & $10,30 \pm 0,20$ & 13,70 & $6,00^{b}$ & 4,00 \\
\hline Lipídeos (\%) & $2,65 \pm 0,06$ & 4,80 & $2,00^{\mathrm{b}}$ & 2,00 \\
\hline Carboidratos $(\%)$ & $53,63 \pm 0,28$ & 17,90 & $60,00^{\mathrm{b}}$ & 10,00 \\
\hline Calorias (kcal) & $279,73 \pm 1,10$ & 13,90 & $278,00^{\mathrm{b}}$ & 7,00 \\
\hline \multicolumn{5}{|l|}{$\mathbf{P b 3}$} \\
\hline Umidade (\%) & $28,92 \pm 0,44$ & - & $26,00^{\mathrm{a}}$ & - \\
\hline Cinzas $(\%)$ & $1,89 \pm 0,07$ & - & $1,50^{\mathrm{a}}$ & - \\
\hline Proteínas (\%) & $11,33 \pm 0,56$ & 15,10 & $8,20^{b}$ & 7,00 \\
\hline Lipídeos (\%) & $2,62 \pm 0,17$ & 4,76 & $0,00^{\mathrm{b}}$ & 0,00 \\
\hline Carboidratos $(\%)$ & $55,24 \pm 0,08$ & 18,41 & $63,30^{\mathrm{b}}$ & 13,00 \\
\hline Calorias (kcal) & $289,80 \pm 1,22$ & 14,49 & $291,70^{\mathrm{b}}$ & 9,00 \\
\hline \multicolumn{5}{|l|}{ Pi1 } \\
\hline Umidade (\%) & $37,86 \pm 0,28$ & - & $35,00^{\mathrm{a}}$ & - \\
\hline Cinzas $(\%)$ & $2,43 \pm 0,21$ & - & $2,30^{\mathrm{a}}$ & - \\
\hline Proteínas $(\%)$ & $12,06 \pm 0,45$ & 16,08 & $9,40^{\mathrm{b}}$ & 6,00 \\
\hline Lipídeos (\%) & $1,24 \pm 0,14$ & 2,25 & $1,83^{b}$ & 2,00 \\
\hline Carboidratos $(\%)$ & $46,36 \pm 0,28$ & 15,45 & $46,00^{\mathrm{b}}$ & 8,00 \\
\hline Calorias (kcal) & $244,86 \pm 2,08$ & 12,24 & $230,00^{\mathrm{b}}$ & 6,00 \\
\hline \multicolumn{5}{|l|}{$\mathbf{P i 2}$} \\
\hline Umidade (\%) & $34,40 \pm 0,07$ & - & $35,00^{\mathrm{a}}$ & - \\
\hline Cinzas $(\%)$ & $2,44 \pm 0,04$ & - & $2,30^{\mathrm{a}}$ & - \\
\hline Proteínas (\%) & $11,36 \pm 0,05$ & 15,15 & $7,10^{b}$ & 9,00 \\
\hline Lipídeos (\%) & $2,48 \pm 0,05$ & 4,51 & $4,30^{\mathrm{b}}$ & 2,00 \\
\hline Carboidratos $(\%)$ & $49,65 \pm 0,35$ & 16,55 & $5,70^{b}$ & 10,00 \\
\hline Calorias (kcal) & $263,45 \pm 1,76$ & 13,17 & $278,60^{\mathrm{b}}$ & 9,00 \\
\hline \multicolumn{5}{|l|}{$\mathbf{P i 3}$} \\
\hline Umidade (\%) & $36,89 \pm 2,30$ & - & $35,00^{\mathrm{a}}$ & - \\
\hline Cinzas $(\%)$ & $1,92 \pm 0,03$ & - & $2,30^{\mathrm{a}}$ & - \\
\hline Proteínas (\%) & $12,63 \pm 0,50$ & 16,84 & $9,60^{\mathrm{b}}$ & 6,00 \\
\hline Lipídeos (\%) & $1,38 \pm 0,04$ & 2,25 & $0,00^{\mathrm{b}}$ & 0,00 \\
\hline Carboidratos $(\%)$ & $44,02 \pm 1,80$ & 14,67 & $48,00^{\mathrm{b}}$ & 8,00 \\
\hline Calorias (kcal) & $240,00 \pm 5,50$ & 12,00 & $238,00^{\mathrm{b}}$ & 6,00 \\
\hline
\end{tabular}

${ }^{\mathrm{a}}$ Valor disponível na Tabela de Composição Química de Alimentos (TACO, 2011); ${ }^{\mathrm{b}}$ Valor declarado na tabela de informação nutricional do produto; *VD (\%): percentual de Valor Diário Recomendado com base em uma dieta de $2.000 \mathrm{kcal} / \mathrm{dia}$ (DRI, 2005); Fonte: os autores.

Os valores de umidade e cinzas obtidos em análise mostraram-se bastante próximos aos valores disponíveis na Tabela de Composição Química de Alimentos (TACO, 2011). Comparando-se os valores obtidos em análises com aqueles declarados na tabela de informação nutricional dos produtos, observa-se que para a amostra $\mathrm{Pb} 1$, somente o teor de calorias foi maior, enquanto que os resultados de proteínas, lipídios e carboidratos foram menores.

Para a amostra Pi1, verificou-se que os valores de proteínas e de calorias obtidos nas análises apresentaram-se maiores que aqueles declarados na tabela de informação nutricional do produto. Porém, os valores de lipídeos mostraramse inferiores. Nesse caso, a análise físico-química 
de lipídeos favoreceria a informação nutricional do Pi1 e, também, sua compra, já que um conteúdo de lipídeos baixo é, em geral, preferido pelos consumidores. $\mathrm{O}$ mesmo pode ser observado acerca da amostra Pi2 que apresentou teor de lipídeos inferior ao valor declarado na tabela de informação nutricional. Já na amostra Pi3, observou-se que os carboidratos tiveram valores menores nas análises laboratoriais que aqueles declarados na tabela de informação nutricional. Corroborando com os resultados verificados no presente estudo, Lobanco et al. (2009) ao analisarem rótulos nutricionais de alimentos industrializados salgados e doces, habitualmente consumidos por crianças e adolescentes, também observaram que todas as amostras analisadas apresentaram inconformidades com os valores das tabelas de informação nutricional. Similarmente, Barros (2003) identificou irregularidades nas informações nutricionais de leites longa vida em até $57 \%$ das marcas analisadas. Oliveira et al. (2005), ao analisarem linguiças de frango e de pernil também observaram diferenças entre os valores obtidos em análises e os valores declarados nas tabelas de informação nutricional. Sauverbronn (2003), em pesquisa com diferentes marcas de massas alimentícias verificou que os valores de proteínas, gorduras e fibras obtidos em análises, apresentaram diferenças quando comparados aos valores declarados nas tabelas de informação nutricional.

Todas as amostras apresentaram um percentual de valor diário de referência maior que os declarados nas tabelas de informação nutricional, o que poderia prejudicar a interpretação e a decisão de compra do consumidor. De forma semelhante, Grandi e Rossi (2010) avaliando vários aspectos das informações nutricionais de bebidas lácteas fermentadas e iogurtes, observaram que cerca de $20 \%$ dos iogurtes e $50 \%$ das bebidas fermentadas não apresentavam percentual de valores diários em conformidade com os valores reais medidos através de análises físico-químicas. Na Figura 1 estão descritas as porcentagens dos parâmetros analisados em laboratório que mostraram diferença do rótulo dos produtos.

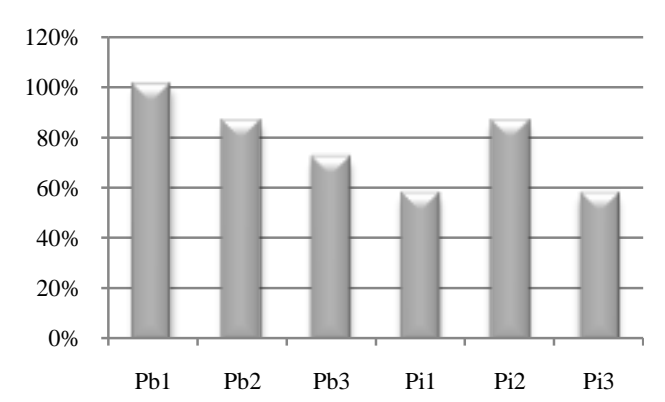

Figura 1 - Porcentagem dos parâmetros analisados que mostraram diferença do rótulo dos pães brancos $(\mathrm{Pb})$ e integrais (Pi). Fonte: os autores.

As amostras $\mathrm{Pb} 1, \mathrm{~Pb} 2$ e $\mathrm{Pi} 2$ foram as que mais apresentaram avaliações laboratoriais diferentes daquelas verificadas nos rótulos $(100 \%$, $85,7 \%$ e $85,7 \%$, respectivamente). Entretanto, as demais amostras também mostraram altas porcentagens de inconformidades com o rótulo, sendo a amostra $\mathrm{Pb} 3$ com 71,4\% e Pi1 e Pi3 com $57,1 \%$. Na Tabela 2 está descrita a porcentagem de inadequações encontradas em relação aos 
valores apresentados nos rótulos das amostras de pães.

Tabela 2 - Porcentagem de adequação entre os valores obtidos em análises laboratoriais e aqueles declarados na tabela de informação nutricional e/ou tabela de composição química de alimentos dos pães brancos (Pb) e integrais (Pi)

\begin{tabular}{lcccccc}
\hline Nutriente & Pb1 & Pb2 & Pb3 & Pi1 & Pi2 & Pi3 \\
\hline${\text { Umidade }(\%)^{\mathrm{a}}}_{\text {Cinzas }(\%)^{\mathrm{a}}}$ & $+31,8^{*}$ & $+21,2^{*}$ & $+11,2$ & $+8,2$ & $-1,7$ & $+5,4$ \\
Proteínas $(\%)^{\mathrm{b}}$ & $+22,0^{*}$ & $+24,0^{*}$ & $+26,0^{*}$ & $+5,6$ & $+6,0$ & $-16,6$ \\
${\text { Lipídios }(\%)^{\mathrm{b}}}_{\text {Carboidratos }(\%)^{\mathrm{b}}}$ & $-12,9$ & $+71,6^{*}$ & $+38,1^{*}$ & $+28,3^{*}$ & $+60,0^{*}$ & $+31,5^{*}$ \\
${\text { Calorias }(\%)^{\mathrm{b}}}^{\text {a }}$ & $-38,0^{*}$ & $+32,5^{*}$ & $+100,0^{*}$ & $-32,2^{*}$ & $-42,4^{*}$ & $+100,0^{*}$ \\
& $-41,5^{*}$ & $-10,6$ & $-12,8$ & $+0,7$ & $+771,0^{*}$ & $-8,3$ \\
\end{tabular}

${ }^{a}$ Valores comparados com a tabela de composição química de alimentos (TACO, 2011); ${ }^{b}$ Valores comparados com a tabela de informação nutricional do produto; *Porcentagem fora da tolerância de 20\% (para mais ou para menos) (BRASIL, 2003); Fonte: os autores.

Considerando-se a tolerância admitida de $20 \%$ (para mais ou para menos) pela ANVISA (BRASIL, 2003), foi observado que das 12 análises realizadas para umidade e cinzas (2 nutrientes de 6 amostras), 5 mostraram-se inadequadas. Destaca-se que, apesar da informação de umidade e cinzas não serem disponibilizadas na informação nutricional dos produtos, estas devem ser consideradas pelas indústrias, uma vez que demonstram características nutricionais importantes dos alimentos aos consumidores. Para os demais nutrientes, proteínas, lipídios, carboidratos e calorias, foi verificado que das 24 avaliações realizadas (4 nutrientes de 6 amostras), somente 10 delas mostraram-se adequadas. Para os lipídeos, todas as amostras estavam fora da tolerância. Resultados similares foram constatados por Câmara et al. (2008), em que verificou-se elevada frequência do descumprimento à legislação brasileira de rotulagem nutricional.

Foi observada elevada variação de adequação entre as amostras analisadas, sendo a menor de $0,6 \%(\mathrm{~Pb} 2)$ e a maior de $771 \%(\mathrm{Pi} 2)$. Para a amostra $\mathrm{Pb} 1$, somente o valor de proteína declarado na tabela de informação nutricional do produto mostrou-se dentro da tolerância. Já para as amostras $\mathrm{Pb} 2, \mathrm{~Pb} 3$ e $\mathrm{Pi} 1$, os valores de carboidratos e de calorias mostraram-se adequados ao limite tolerado. Na Figura 2 está apresentada a porcentagem de adequação (BRASIL, 2003) de cada amostra avaliada em laboratório, quanto aos nutrientes, quando comparada aos valores declarados na tabela de informação nutricional do produto.

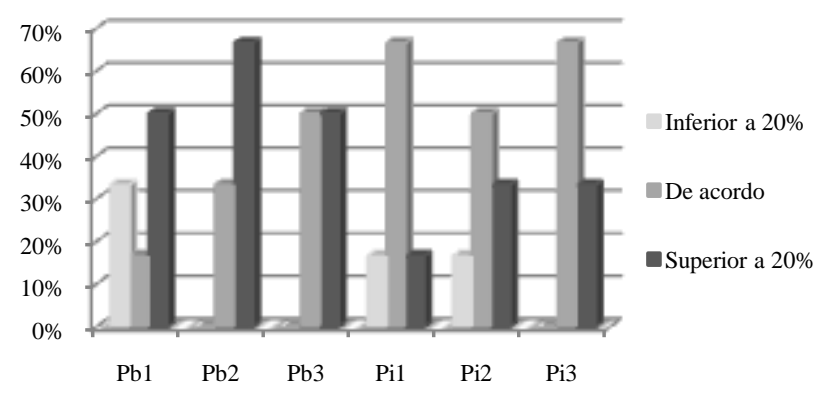

Figura 2 - Porcentagem de adequação (BRASIL, 2003) das amostras analisadas em laboratório, em relação aos nutrientes, comparadas aos valores declarados na tabela de informação nutricional dos pães brancos $(\mathrm{Pb})$ e integrais (Pi). Fonte: os autores. 
As amostras de pão branco obtiveram, de um modo geral, maior número de nutrientes com inadequações (inferior e/ou superior) à legislação (BRASIL, 2003), quando comparadas às amostras de pão integral. Porém, 100\% dos produtos analisados apresentaram algum tipo de inadequação em relação à legislação (BRASIL, 2003). Resultados semelhantes foram verificados por Silva e Ferreira (2010), que avaliaram a composição química de queijo minas frescal, queijo minas frescal "light" e ricota, onde 92\% das marcas analisadas apresentaram inadequação superior a $20 \%$ para algum tipo de nutriente. Também, Esper (2006) analisando 15 diferentes marcas de ricota, constataram que $93 \%$ apresentavam algum parâmetro fora da variação de $\pm 20 \%$ admitido pela legislação.

Atualmente, existe uma tendência geral por parte dos consumidores em conhecer o valor nutricional dos alimentos industrializados, principalmente, aqueles nutrientes que possam trazer algum tipo de benefício e/ou prejuízo ao organismo (GRANDI; ROSSI, 2010). Dessa forma, a disponibilidade de corretas informações nutricionais nos rótulos dos produtos são imprescindíveis, sendo cada vez mais observadas pela população. Esse fato foi confirmado por Coutinho (2004), ao entrevistar consumidores brasileiros, sendo que $43 \%$ deles procuravam obter informações nutricionais no rótulo dos alimentos no momento da compra. Principalmente com o objetivo de prevenir ou controlar doenças relacionadas à alimentação, como hipertensão arterial, diabetes, doenças cardíacas e obesidade.

\section{CONCLUSÃO}

A maioria dos resultados verificados nas análises laboratoriais dos pães brancos e integrais foram superiores àqueles apresentados nos rótulos dos produtos e na tabela de composição química de alimentos. Esse fato pode causar prejuízos ao consumidor, tanto econômico como nutricional, uma vez que a ingestão de nutrientes em quantidades inadequadas poderá elevar o risco de doenças.

As amostras de pão branco foram as que apresentaram maiores quantidades de inadequações nos parâmetros analisados e comparados com a legislação brasileira vigente. Dessa forma, torna-se indispensável aos fabricantes e indústrias um maior investimento nesta área, já que é direito do consumidor conhecer a composição do alimento que está adquirindo.

\section{REFERÊNCIAS}

ATWATER, W.O.; WOODS, C.D. The chemical composition of American food materials. Farmers' Bulletin, n.28, U.S. Department of Agriculture. Washington, 1896. 46p.

BARROS, D.L.G. Qualidade de leite UHT comercializado em Brasília-DF. 2003. Dissertação (Mestrado em Nutrição Humana) Faculdade de Ciências da Saúde, Universidade de Brasília, Brasília, 2003.

BENDINO, N.I.; POPOLIM, W.D.; OLIVEIRA, C.R.Á. Avaliação do conhecimento e dificuldades de consumidores frequentadores de supermercado convencional em relação à rotulagem de alimentos e informação nutricional. The Journal of Health Sciences Institute, v.30, n.3, p.261-25, 2012.

BLIGH, E.G.; DYER, W.J. A rapid method of total lipid extraction and purification. Canadian 
Journal of Biochemistry Physiology, v.37, n.8, p.911-917, 1959.

BRASIL. Ministério da Saúde. Agência Nacional de Vigilância Sanitária. ANVISA. RDC no 360:

Dispõe sobre regulamento de rótulos de alimentos, de 23 de dezembro de 2003.

Disponível em: http://portal.anvisa.gov.br/wps/ wcm/connect/ec3966804ac02cf1962abfa337abae 9

d/Resolucao_RDC_n_360de_23_de_dezembro_d e_2003.pdf?MOD=AJPERES $>$. Acesso em: 14 out. 2017.

CÂMARA, M.C.C.; MARINHO, C.L.C.; GUILAM, M.C.; BAGA, A.M.C.B. A produção acadêmica sobre rotulagem de alimentos no Brasil. Revista Panamericana de Salud Pública, v.23, n.1, p.52-58, 2008.

COUTINHO, J.G. Estabelecimento de alegação de saúde nos rótulos de alimentos e bebidas embalados. 2004. Dissertação (Mestrado em Nutrição Humana) - Faculdade de Ciências da Saúde, Universidade de Brasília, Brasília, 2004.

DIETARY REFERENCE INTAKES (DRI).

Dietary Reference Intakes for energy, carbohydrate, fiber, fat, fatty acids, cholesterol, protein and amino acids. Washington, D.C.: The National Academies Press, 2005. 1331p.

ESPER, L.M.R. Diagnóstico da qualidade de ricotas comercializadas no Município de Campinas-SP. 2006. Dissertação (Mestrado em Tecnologia de Alimentos) - Faculdade de Engenharia de Alimentos Campinas, Universidade Estadual de Campinas, Campinas, 2006.

GRANDI, A.Z.; ROSSI, D.A. Avaliação dos itens obrigatórios na rotulagem nutricional de produtos lácteos fermentados. Revista do Instituto Adolfo Lutz, v.69, n.1, p.62-68, 2010.

INSTITUTO ADOLFO LUTZ. Normas analíticas do Instituto Adolfo Lutz: Métodos químicos e físicos para análises de alimentos. v.1. 4 ed. Brasília: IAL, 2008. 1002p.

LOBANCO, C.M.; VEDONATO, G.M.; CANO, C.B.; BASTOS, D.H.M. Fidedignidade de rótulos de alimentos comercializados no município de São Paulo, SP. Revista de Saúde Pública, v.43, n.3, p.499-505, 2009.
MENEZES, E.W.; GIUNTINI, E.B.; DAN, M.C.T.; LAJOLO, F.M. New information on carbohydrates in the Brazilian Food Composition Database. Journal of Food Composition and Analysis, v.22, n.5, p.446-452, 2009.

OLIVEIRA, M.J.; ARAÚJO, W.M.C.; BORGO, L.A. Quantificação de nitrato e nitrito em linguiças do tipo frescal. Ciência e Tecnologia de Alimentos, v.25, n.4, p.737-742, 2005.

SAUVERBRONN, A.L.A. Análise laboratorial da composição de alimentos processados como contribuição ao estudo da rotulagem nutricional obrigatória de alimentos e bebidas embalados no Brasil. 2003. Dissertação (Mestrado em Vigilância Sanitária de Produto) - Fundação Instituto Oswaldo Cruz, Rio de Janeiro, 2003.

SILVA, L.F.M.; FERREIRA, K.S. Avaliação de rotulagem nutricional, composição química e valor energético de queijo minas frescal, queijo minas frescal "light" e ricota. Alimentos e

Nutrição, v.21, n.3, p.437-41, 2010.

TACO. Tabela Brasileira de Composição dos alimentos. UNICAMP. 4. ed. Campinas: NEPA, 2011. 161p.

\section{Jocelaine Dall'agnol}

Nutricionista, Graduada pela Universidade Estadual do Centro-Oeste.

Liégi Tajana Ferranti

Nutricionista, Graduada pela Universidade Estadual do Centro-Oeste.

Letícia Claudy

Nutricionista, Graduada pela Universidade Estadual do Centro-Oeste.

\section{Gabriel Cecchin}

Engenheiro de Alimentos, Graduado pela Universidade Estadual do Centro-Oeste.

\section{Herta Stutz Dalla Santa}

Bióloga, Doutora. Docente do Curso de Engenharia de Alimentos da Universidade Estadual do CentroOeste.

\section{Bruna Menegassi}

Nutricionista, Mestre. Docente do Curso de Nutrição da Universidade Estadual do Centro-Oeste.

Daiana Novello
Nutricionista, Doutora. Docente do Curso de
Nutrição e do Mestrado Interdisciplinar em
Desenvolvimento Comunitário da Universidade
Estadual do Centro-Oeste.

